\title{
Correction to: The quadriceps active ratio: a dynamic MRI-based assessment of patellar height
}

\author{
Sergio Barroso Rosa ${ }^{1,2} \cdot$ Zaid Bahho $^{1} \cdot$ Kenji Doma $^{1} \cdot$ Kaushik Hazratwala $^{1} \cdot$ Peter McEwen ${ }^{1} \cdot$ Varaguna Manoharan $^{1}$. \\ Brent Matthews ${ }^{1} \cdot$ Matthew Wilkinson $^{1}$
}

Published online: 31 March 2018

(c) Springer-Verlag France SAS, part of Springer Nature 2018

\section{Correction to: \\ European Journal of Orthopaedic Surgery \& Traumatology https://doi.org/10.1007/s00590-018-2170-6}

In the online version of the original article, one of the white lines was not accurately drawn in Fig. 1.

The revised Fig. 1 is placed here:

The original article can be found online at https://doi.org/10.1007/ s00590-018-2170-6.

Sergio Barroso Rosa

dr.sbarroso@gmail.com

Zaid Bahho

zaid_bahho@hotmail.com

Kenji Doma

kenji.doma@jcu.edu.au

Kaushik Hazratwala

koshman1@bigpond.com

Peter McEwen

peter@kneesurgeon.com.au

Varaguna Manoharan

vmanoharan86@gmail.com

Brent Matthews

drbrentmatthews@gmail.com

Matthew Wilkinson

mprwilkinson@hotmail.com

1 The ORIQL (Orthopaedic Research Institute of Queensland), 7 Tuner Street, Pimlico, QLD 4812, Australia

2 Univeristy of Las Palmas de Gran Canaria, Las Palmas,

Canary Islands, Spain

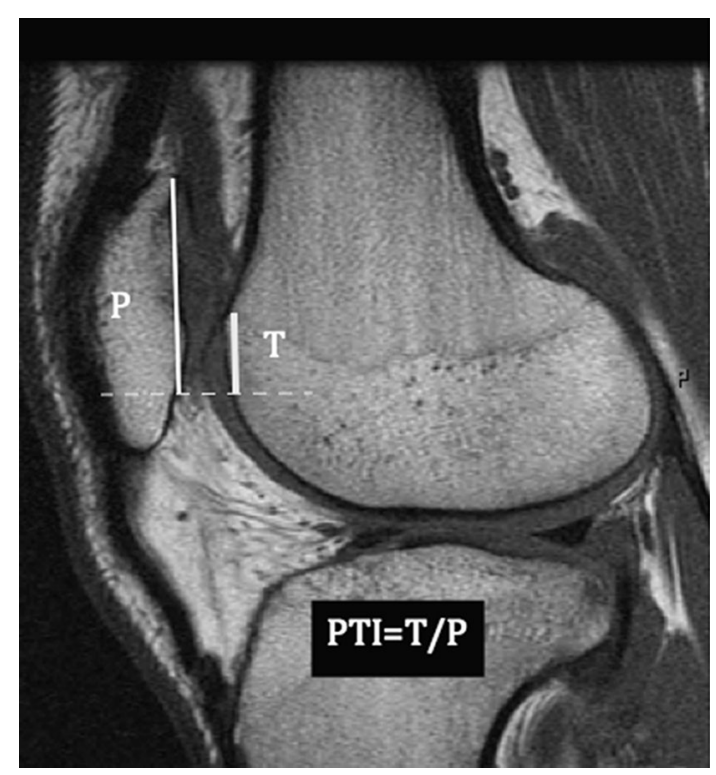

Fig. 1 Calculation of the PTI-patellotrochlear index (quads active ratio if assessed under active contraction of the quadriceps muscles) 